\title{
THE EFFECT OF DELAYED SUCKLING ON MAINTENANCE OF MATERNAL RESPONSIVENESS AND SELECTIVITY OF BARKI SHEEP
}

\author{
A.Y. Abdel- Moneim, M.S. Abd-Alla and G.M. Ashmawi \\ Department of Animal Production, Faculty of Agriculture, Cairo University, Giza, \\ Egypt
}

\section{SUMMARY}

Twenty-five Barki ewes were divided into 5 equal groups. In the first treatment $\left(T_{1}\right)$ (control treatment) lamb stayed with its mother for only one-hour immediately after lambing. In the second treatment $\left(T_{2}\right)$ (separation treatment), lamb was isolated from its mother immediately after birth for one-hour. In the third treatment $\left(T_{3}\right)$ (udder coverage treatment), lamb stayed with its mother for one-hour immediately after birth but it was prevented from suckling by using a "Package". In the fourth treatment $\left(T_{4}\right)$ (Grafting with alien lamb treatment), young was separated from its mother for one-hour immediately after birth and was bottle - fed the milk of its own mother. During separation period, the mother nursed the alien lamb from its udder. In the fifth treatment $\left(T_{5}\right)$ (fostering treatment), the lamb was separated from its mother for one-hour immediately after birth and it was naturally suckled from the udder of alien ewe during the separation period.

Early post - partum separation between ewe and lamb led to break of motheryoung ties. The different senses (visual, olfactory and auditory cues) during the first hour after birth were important to establish mother-young bond even when prevented from suckling for 1-h after birth. The presence of alien lamb with dam immediately after parturition may allow the dam to nurse alien lamb. Fostering lambs on alien dams during the separation period immediately after birth did not enhance the acceptability with own mothers when re-turned to them .

Keywords : Maternal behaviour, Barki ewes, package, grafting, fostering

\section{INTRODUCTION}

Bahaviour of both the dam and offspring during the period surrounding parturition is critical, as failure to form a mother - offspring relationship can result in poor growth or death. Therefore, proper maternal behaviour at parturition is essential to minimize the detrimental effects of environmental factors. Taskin et al. (1996) showed that the identification of factors controlling the establishment of the mother-young attachment can lead to the development of new methods of fostering and provide sound guidelines for selection. The objective of the present study is to investigate the behavioural responsiveness of ewe and lamb to separation, udder coverage, grafting and fostering.

Issued by The Egyptian Society of Animal Production 


\section{MATERIALS AND METHODS}

\section{A. Animals :}

Twenty-five Barki ewes (15 multiparous and 10 primiparous ewes) and their lambs as well as 5 Barki alien lambs born to other Barki ewes within the same time were used. Animals belonged to the Small Ruminant Experimental Farm of the Faculty of Agriculture, Cairo University, Giza, Egypt . The primiparous ewes were nearly 1.5 years old while the multiparous ones aged 2-4 years. Body weight of primiparous and multiparous Barki ewes used averaged $40.5 \mathrm{~kg}$ and $43.4 \mathrm{~kg}$, respectively. The 25 Barki ewes were divided into 5 equal groups. Each group contained 3 multiparous and 2 primiparous ewes. All animals were in a good healthy condition throughout the experimental period. All experiments were carried out during the natural parturition season in autumn 2001.

\section{B. Housing :}

Seven days before the expected date of parturition, ewes were isolated from the main herd of pregnant ewes and were housed in a half-shaded pen $12.9 \times 8.2$ meter with a 1.6 meter high fence and a 3.5 meter high roof (waiting pen). The pen had a concrete floor covered with deep bedding of rice straw. The pen had two built in 660 x 48 × 35 centimeter troughs, one trough was used for forages and the other for concentrates. Water supply was available all the time for the animals in the pen.

One week before starting the experiments, a video camera (Hitachi VM-E 338 E, $8 \mathrm{~mm}, \quad$ X 150 Digital Zoom, Digital Signal processing "D.S.P." and Wide Angle Lens, Tokyo, Japan) was fixed 1.20 meter high in the waiting pen.

A test pen, 9.9 x 8.2 meter, with a 1.6 meter high fence and a 3.5 meter high roof was used. This pen had clean concrete floor without any bedding. The pen was sunny and far from any source of disturbance. At a corner of the test pen, three adjacent delivery cages made from wire mesh , $1.2 \times 1.2$ meter each, with 1.25 meter high fence and a wire mesh door. These delivery cages were used instead of lambing hurdles as they allowed the observer to watch clearly the observed mother with its young at and after parturition without any disturbance. All observations were recorded using the video camera. This video camera was fixed 1.2 meter high and 2.5 meter away from the delivery cage. Meanwhile , the video camera was equipped with a Zoom lens so that the observer could stay outside the test pen (behind the gate of the test pen) some 8.0 meter from the delivery cages. In the meantime, a mini T.V. (Greatrodo "7" Portable B/W, No. GR T.V.- 708, China) was used to allow having a clear picture during recording and for the reproduction (replay) of the images . Chronometer was used to measure time after recording period.

During parturition period, ewes were exposed to normal daylight, while a fluorescent lamp (TL $40 \mathrm{~W} / 54$ ) was used by night to illuminate the delivery cages and to give sufficient light for clear recording by the camera.

\section{Feeding:}

Ewes were fed ad libitum on Egyptian clover (Trifolium alexandrinum) from November to April while from May to September, they were given liberal amount of Darawa (Zea maize L.) or sugar corn (Sorghum vulgare var. succharatum, Boeral), in addition to clover hay. Each ewe was supplemented daily with 800 grams of a 
concentrate mixture .The concentrate mixture consisted of $30 \%$ decorticated cotton seed meal, 30\% wheat bran , 30\% yellow corn, 5\% rice bran (in stead of molasses), $3 \%$ limestone, $1 \%$ sodium chloride and $1 \%$ common salt. Furthermore, salt blocks were available for the animals all the time.

\section{Experimental procedures::}

The first treatment (T1) (control treatment), the lamb stayed with its mother for one-hour immediately after lambing, then the lamb was separated from its mother for 15 min with the intention of inducing rapid suckling when returned back to mother. The response of the young to suckle treatment was tested for $5 \mathrm{~min}$ in a $1.2 \mathrm{~m}^{2}$ delivery cage with wire mesh door. The behavioural acts manifested by both mother and lamb were recorded. The second treatment (T2) (separation treatment), aimed at investigating the effect of separation between ewe and lamb on mother-young relationship and behaviour during the early period of life of the lamb. The lamb was isolated from its mother immediately after birth for one-hour. Thus no ewe-lamb contact what so ever took place. The lamb was put in a piece of clean cloth and was carried to another pen far from its mother to prevent hearing, smelling and licking by the dam (Ramirez et al., 1996b). During this period, the lamb was twice bottle-fed the milk of its mother, the first one after $30 \mathrm{~min}$ and the second during the next 30min of isolation. The lamb was kept away from its mother for another $15 \mathrm{~min}$ but without suckling any milk, to stimulate suckling desire. Lamb was introduced to its mother in a $1.2 \times 1.2$ meter delivery cage (test cage) for $5 \mathrm{~min}$ and the behavioural acts displayed by mother and young were observed. In the third treatment (T3) (udder coverage treatment), the lamb stayed with its mother for one-hour immediately after birth but the lamb was prevented from suckling as the udder and teats were completely bagged up with a thick piece of cloth. So there was direct visual, olfactory and auditory contact without suckling. The young lamb was separated from its mother for further $15 \mathrm{~min}$ by keeping it out of the pen. Immediately following such isolation period, the lamb was put again with its mother for 5 min test period to study the influence of prevention of suckling immediately after birth on relationship between mother and young when direct contact between mother and young took place. In the fourth treatment (T4) (grafting treatment with alien lambs), the young was separated from its mother for one-hour immediately after birth. The lamb was bottle-fed the milk of its own mother. During separation period, the mother nursed the alien lamb from its udder. Afterwards, own and grafted alien lambs were separated for 15 min without contact. At the end of this period, own lamb was tested with its mother and alien lamb was tested with the foster mother for $5 \mathrm{~min}$ individually in a test cage to investigate the effect of presence of alien lamb with dam immediately after parturition in the absence of own lamb on mother-young attachment. In the fifth treatment (T5) (fostering treatment), the lamb was separated from its mother for one-hour immediately after birth. During separation period, the lamb was naturally suckled from the udder of alien ewe. After this time, the lamb was isolated from the alien ewe without suckling for $15 \mathrm{~min}$. Thereafter, the lamb was tested with its own mother for $5 \mathrm{~min}$ in a test cage to study the effect of fostering lamb on alien dam during the separation period after birth on mother-young bond . 
The maternal and neonatal parameters which were recorded for each mother and the offspring for $5 \mathrm{~min}$ following parturition using video tape were measured according to (Levy et al., 1991) as follows:

\section{a) Positive maternal behaviour :}

1 - Latency to first suckling .

$$
(\min )
$$

$(\min )$

3 - Number of acceptance at the udder during suckling attempts .

(count)

(count)

b) Negative maternal behaviour:

1 - Number of high-pitched bleats.

2- Number of rejections at the udder during suckling attempts.

3- Number of threats and head butts directed towards the lamb.

The first four parameters are indicative of acceptance of the lamb, while the last three parameters indicate lamb rejection.

\section{E. Statistical Analysis of Data:}

The effect of delayed suckling on maternal responsiveness of Barki ewes was evaluated using the GLM procedure for repeated measures analysis of variance (SAS, 1995 ). All effects were assumed fixed except the random error, which was assumed normally and independently distributed with mean 0 and variance $\sigma^{2}$. Duncan's New Multiple Range Test was applied to test the significant differences among means of different treatments.

\section{RESULTS AND DISCUSSION}

It should be pointed out that three of five separated lambs $\left(\mathrm{T}_{2}\right)$ did not show latency to first suckling, suckling time or number of acceptance at the udder during suckling attempts. The other two separated lambs (Table 1) took longer time to first suckling, spent less suckling time and were less accepted at the udder than the nonseparated lambs (control treatment) $\left(\mathrm{T}_{1}\right)$. Meanwhile, separated dams emitted lower rate of low-pitched bleats. Differences due to separation in all previous displays were non-significant (Table 1). Hence, it seemed that separation attenuated the positive maternal behaviour of Barki ewes. In the meantime, separated ewes $\left(\mathrm{T}_{2}\right)$ emitted less high-pitched bleats and made less number of rejections to suckling attempts than nonseparated ones $\left(T_{1}\right)$, apparently because they made comparatively higher number of threats and head butts towards the lambs (Table 1). It could be concluded that the immediate and early post-natal separation between ewe and lamb negatively affects mother-young ties. The obtained results are in harmony with those obtained by Collias (1956), Smith et al. (1966) and Poindron et al. (1979), who reported that removal of the newborn at parturition led to the fading of maternal responsiveness within $12 \mathrm{~h}$ in most animals. Meanwhile, Porter et al. (1995) showed that when the lambs were removed from their mothers immediately after birth and were tested in isolation, lambs emitted more distress bleats than they did when paired 


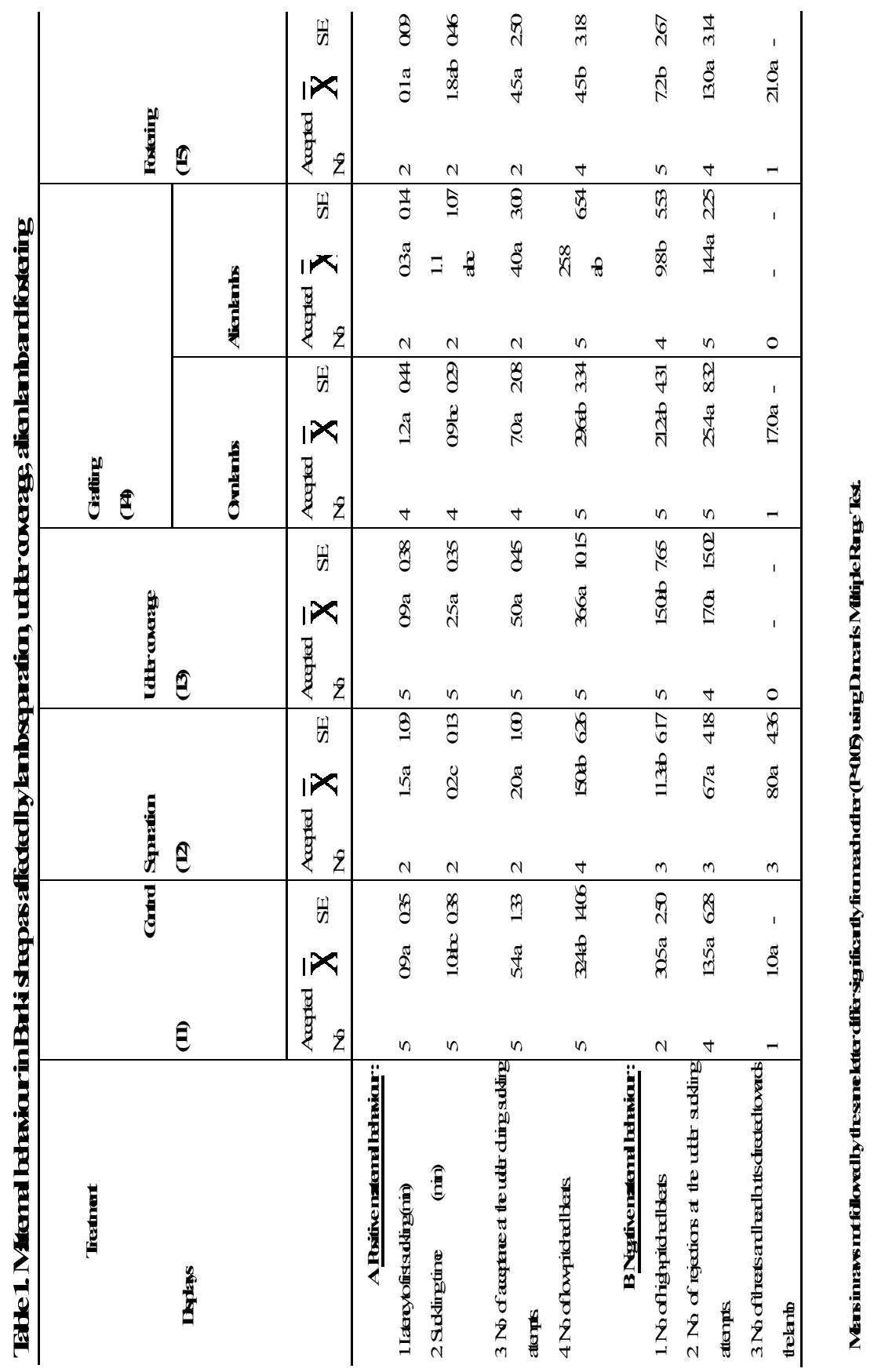


with a social partner-either their twin $(\mathrm{P}<0.01)$ or an unfamiliar lamb $(\mathrm{P}<0.05)$. Furthermore, Nowak et al. (1997) found that preventing the lamb from suckling for $6 \mathrm{~h}$ in the first day after birth affected the development of the ewe-lamb relationship and resulted inability of the lamb to show any preference towards its dam. On the other hand, in Murciano-Granadina goats, Ramirez et al. (1996a,b) observed that after immediate post-partum separation of does from their kids for one hour, no maternal response was maintained after 1-h separation.

Previous studies on sheep have shown that while both dam and offspring depend on olfactory cue for mutual recognition at close quarters, recognition at a distance is dependent on visual and auditory cues (Lindsay and Fletcher, 1968; Poindron and Carrick, 1976; Alexander, 1977 and Alexander and Shillito, 1977). In the present study animals were kept in pens. Hence, there is no denying for the importance of olfaction for a Barki mother in recognizing its lamb. Lamb odour results from "maternal labelling" particularly by the intake of mothers milk, and partially by licking (grooming) just after birth. Hence, it would be logical to assume that prevention of suckling, by udder coverage, would lead to lower rate of maternal and neonatal behaviours. Our observations showed that all ewes in the udder coverage treatment $\left(\mathrm{T}_{3}\right)$ easily recognized its lambs. In addition, no significant difference existed in any of the studied displays between intact $\left(\mathrm{T}_{1}\right)$ and udder covered ewes $\left(T_{3}\right)$. Moreover, ewes with covered udders $\left(T_{3}\right)$ did not perform threats or head butts towards their lambs (Table 1). The obtained results contradict with those of Gonyou and Stooky (1987) who showed that a ewe relies heavily on odour cue to identify its own lamb, and those of Levy et al. (1991) who emphasized that the recognition of Ilede-France lambs could result from their mothers' milk.

It should be pointed out that the period during which udders were covered, was relatively short and was not enough to cause the expected effect. However, specific tests of varying periods of udder coverage are required to confirm that olfactory cue is implicated in mother-lamb bond.

The influence of grafting an alien lamb $\left(\mathrm{T}_{4}\right)$ immediately after birth on maternal behavioural responses as well as how ewe responds to re-union with its own lamb were investigated. Only two out of the five ewes tested accepted the alien lambs $\left(\mathrm{T}_{4}\right)$. Alien lambs $\left(\mathrm{T}_{4}\right)$ introduced to these two ewes were faster to suckle and suckled for longer time than those having own lambs $\left(\mathrm{T}_{1}\right)$. But they were less accepted at the udders of their mothers and they emitted less number of low-pitched bleats compared to those having their own lambs $\left(\mathrm{T}_{1}\right)$ (control treatment) (Table 1). However, non of the differences, concerning positive maternal behaviour displays, reached a significant level (Table 1). The expression of negative maternal behaviour towards alien lamb was not significantly different between grafted (alien) lambs $\left(\mathrm{T}_{4}\right)$ and those of the control treatment $\left(\mathrm{T}_{1}\right)$ (own lambs). These results may indicate that lambs grafted during the first hour after birth could be accepted by a foster mother. In this context, Bordi et al. (1994) concluded that the maternal selectivity is developed within $4 \mathrm{~h}$ after parturition. The authors found that mother goats could accept own kids and alien ones within $4 \mathrm{~h}$ after parturition.

When own lamb $\left(\mathrm{T}_{4}\right)$ was returned to its mother following removal of the alien lamb, most of the positive maternal behaviour displays, i.e. latency to first suckling, suckling time and number of low-pitched bleats were attenuated compared to those of 
the control treatment $\left(\mathrm{T}_{1}\right)$ (own lamb bonded with mother since birth). But ewes accepted their own lambs $\left(\mathrm{T}_{4}\right)$ more than those of the control treatment $\left(\mathrm{T}_{1}\right)$. On the other side, re-union of own lamb and own mother $\left(\mathrm{T}_{4}\right)$ was accompanied with less rate of high-pitched bleats and greater number of rejections at the udder as well as higher number of threats and head butts (Table 1). Interestingly, no significant difference was found in any of the tested behavioural displays between re-bonded own lambs, and those continuously kept with their mothers since lambing (control group). Hence, it seemed that re-union of mother and own lamb, after grafting of alien lamb within the periods used in the present work, would not affect expression of maternal behaviour. Similar results were reported by Alexander et al. (1983) who studied the effect of washing lambs (own or alien) on maternal acceptance of Merino ewes. They found that, 7 to $47 \%$ of mothers permitted alien washed lambs to suckle and most of those dams were able to distinguish their own washed lambs from alien washed ones. While, 25 to $43 \%$ of mothers refused to allow its own washed lambs to suckle.

The effect of fostering of the lamb on another ewe $\left(\mathrm{T}_{5}\right)$ on maternal behaviour was tested. It was noticed (Table 1) that latency to first suckling, suckling time and number of acceptance at the udder during suckling attempts were not achieved by $60 \%(3 / 5)$ of lambs grafted on alien mother during the first hour after birth. The other two lambs $(2 / 5)$ involved in fostering treatment $\left(\mathrm{T}_{5}\right)$ had less latency to first suckling and less number of acceptance at the udder and longer suckling time than control treatment $\left(T_{1}\right)$. But differences were not significant (Table 1). Meanwhile, foster ewes $\left(\mathrm{T}_{5}\right)$ emitted lower rate of low-pitched bleats (Table 1) than those belonged to the control treatment $\left(\mathrm{T}_{1}\right)$.

On the other hand, grafted lambs $\left(\mathrm{T}_{5}\right)$ were rejected more than own lambs (control treatment) $\left(\mathrm{T}_{1}\right)$. All fostered ewes emitted comparatively lower rate of high-pitched bleats than that of the (control treatment). Whereas, the average number of highpitched bleats emitted by foster ewes was significantly $(\mathrm{P}<0.05)$ lower than that of the (control treatment). In the meantime, a foster mother $\left(\mathrm{T}_{5}\right)$ seemed to threats and butts its lamb exceedingly more than did that of the control treatment $\left(\mathrm{T}_{1}\right)$ (Table 1). The obtained results revealed that grafting lambs during the separation period immediately after birth did not enhance the acceptability of own mothers when returned to their lambs.

On the other side, Table (2) shows no significant differences in different maternal displays due to delayed suckling, except suckling time. Delayed suckling had a significant $(\mathrm{P}<0.05)$ influence on suckling time (Table 2). Meanwhile, the results in Table (1) show that lambs in udder coverage treatment $\left(\mathrm{T}_{3}\right)$ and in fostering treatment $\left(\mathrm{T}_{5}\right)$ had significantly $(\mathrm{P}<0.05)$ higher suckling time than those in the separated treatment $\left(\mathrm{T}_{2}\right)$. This result cast light on the serious effect of early separation on mother-young bond. 
Table 2. Analysis of variance for the effect of lamb separation, udder coverage, alien lamb and fostering on maternal behaviour in Barki sheep Displays Source of variation

\begin{tabular}{cccc}
\hline \multicolumn{2}{c}{ Treatment } & \multicolumn{2}{c}{ Residual } \\
\hline d.f. & M.S. & d.f. & M.S. \\
\hline
\end{tabular}

\section{A. Postive maternal behaviour:}

1. Latency to first suckling

2. Suckling time

3. No. of accept. at the udder during suckling attempts

4. No. of low-pitched bleats.

\section{B. Negative maternal behaviour:}

1. No. of high-pitched bleats.

2. No of rejections at the udder suckling attempts.

3. No. of threats and head butts directed towards the lamb.
5

5

$2.33^{*}$

7.45

14

8.84

0.65

5
627.32

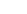

25

\section{$* \mathrm{P}<0.05$}

\section{REFERENCES:}

Alexander, G., 1977. Role of auditory and visual cues in mutual recognition between ewes and lambs in Merino sheep. Appl. Anim. Behav. Sci., 3 : 65-81.

Alexander G. and E.E. Shillito, 1977. The importance of odour, appearance and voice in maternal recognition of the young in Merino sheep (ovis aries). Appl. Anim. Behav. Sci., 3 : 127-135.

Alexander, G. D. Stevens and L.R. Bradly, 1983. Washing lambs and Confinement as aids to fostering. Appl. Anim. Behav. Sci. 10: 251-261.

Bordi, A., G. De-Rosa, F. Napolitano, M. Litterio, V. Marino and R. Rubino, 1994. Post-partum development of the mother-young relationship in goats. Appl. Anim. Behav. Sci., 42:145-152.

Collias, N.E., 1956. The analysis of socialization in sheep and goats . Ecology, $37: 228-239$. 
Gonyou, H.W. and J.M. Stookey, 1987. Maternal and neonatal behaviour. Vet. Clin. North Am. Food Anim. Pract. 3: 231-249.

Levy, F., R. Gervais, U. Kindermann, M. Litterio, P. Poindron and R. Porter, 1991. Effects of early post-partum separation on maintenance of maternal responsiveness and selectivity in parturient ewes. Appl. Anim. Behav. Sci., 31: 101110.

Lindsay, D.R. and I.C. Fletcher, 1968. Sensory involvement in the recognition of lambs by their dams. Anim. Behav., $16: 415-417$.

Nowak, R., T.M. Murphy, D.R Lindsay, P. Alster, R. Anderson and K. UvansMoberg, 1997. Development of a preferential relationship with the mother by the newborn lamb: importance of the suckling activity. Physiol. Behav., 61:681-688.

Poindron, P. and M.J. Carrick, 1976. Hearing recognition of the lamb by its mother. Anim. Behav., 24 : 600-602.

Poindron, P., G.B. Martin and R.D. Hooley, 1979. Effects of lambing induction on the sensitive period for the establishment of maternal behavior in sheep. Physiol. Behav., 23:1081-1087 (Abstr.).

Porter, R.H., R. Nowak, and P. Orgeur, 1995. Influence of a conspecific agemate on distress bleating by lambs. Appl. Anim. Behav. Sci., 45:239-244.

Ramirez, A., A. Quiles, M.L. Hevia, F.Sotillo, and M.C Ramirez, 1996a. Effects of immediate and early post-partum separation on maintenance of maternal responsiveness in parturient multiparous goats. Appl. Anim. Behav. Sci., 48:216224.

Ramirez, A., A. Quiles, M.L. Hevia, F. Sotillo and M.D. Carmen-Ramirez, 1996 b. Influence of forced contact on the maternal-filial bond in the domestic goat after different periods of post-partum separation. Small Rumin. Res. 23:75-81.

SAS, 1995. SAS User's Guide: Statistics. SAS Inst. Inc., Cary, N.C.

Smith F.V., C. Van-Toler and T. Boyes, 1966. The "critical period" in the attachment of lambs and ewes. Anim. Behav., 14 : 120-125.

Taskin, T., M. Kaymakci, A. Karaaslan and D. Arisoy-Basaran, 1996. The important and relationships between mother and lambs . Ege-Universitesi-Ziraat-FakultesiDergisi. 33:199-206. (Abstr.). 
تأثير تأخير الرضاعة على المحافظة على قدرة الإستجابة الأموية والاختيار فى أغنام البرقى

أحمد يحيى عبد المنعم ، ممدوح سيد عبد الله ، جلال الدين محمد عشماوى

قسم الإتتاج الحيوانس، كلية النزاعة، جامعة القاهة، الجيزة ، مصر .

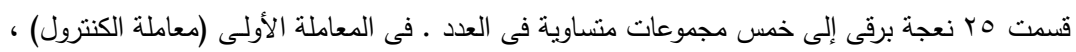

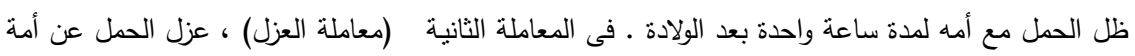

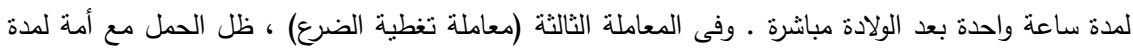

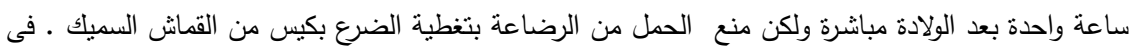

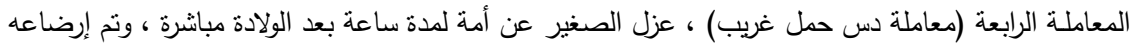

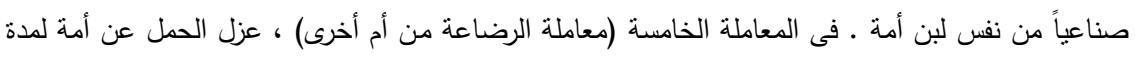

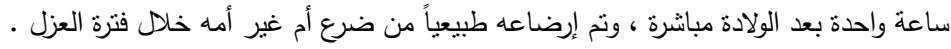

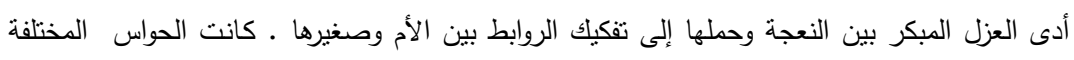

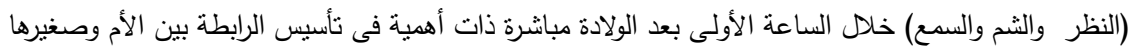

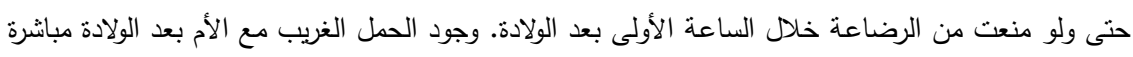

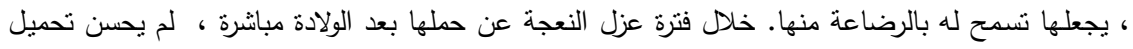

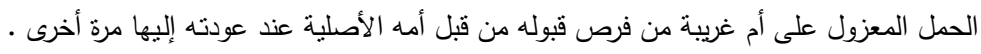

